\title{
A eficácia da mentoria escolar na promoção do desenvolvimento socioemocional e instrumental de jovens*
}

\author{
Francisco Simões \\ Madalena Alarcão \\ Universidade de Coimbra
}

Correspondência:

Francisco Alexandre Melo Simões

Canada da Francesa, 28, São Mateus da Calheta Angra do Heroísmo

9700-551 - Açores, Portugal

E-mail: francisco.simoes@cipp-terceira.com

* Trabalho financiado pela Fundação para a Ciência e Tecnologia do Ministério da Ciência, Tecnologia e Ensino Superior de Portugal (SFRH /BD/60823/2009).

\section{Resumo}

0 presente artigo constitui uma revisão da literatura acerca da eficácia dos programas de mentoria escolar na promoção do desenvolvimento socioemocional e instrumental de jovens. Para tal, procedeu-se a uma comparação entre as investigações empíricas mais significativas sobre o assunto. A partir dessa análise, é possível verificar que os resultados atribuídos a programas de mentoria são contraditórios e, tendencialmente, de magnitude reduzida. Diversas fragilidades metodológicas parecem afectar os resultados obtidos na maior parte dos trabalhos avaliados, entre as quais se destacam o tamanho reduzido das amostras e a inexistência de controlo experimental da variabilidade inerente aos sujeitos. A par das limitações metodológicas, a revisão de literatura efectuada permitiu ainda identificar variações importantes na estruturação dos próprios programas de mentoria escolar e uma ponderação inconsistente de variáveis definidoras de boas práticas. Todos esses factores parecem dificultar a obtenção de conclusões sólidas sobre a eficácia desse tipo de intervenção em meio escolar. A realização de mais estudos, com poder inferencial, sobre programas bem estruturados, um maior cuidado na definição dos planos de investigação e a consideração do professor como figura habilitada ao desempenho cumulativo das funções de mentor, ao contrário do que sucede em programas desenvolvidos em países anglosaxónicos, são algumas das recomendações a seguir em futuras investigações sobre essa temática.

\section{Palavras-chave}

Mentoria escolar - Desenvolvimento socioemocional Desenvolvimento instrumental - Eficácia. 


\title{
The effectiveness of school mentoring in promoting the socioemotional and instrumental development of youngsters*
}

\author{
Francisco Simões \\ Madalena Alarcão \\ University of Coimbra
}

\author{
Contact: \\ Francisco Alexandre Melo Simões \\ Canada da Francesa, 28, São Mateus da Calheta \\ Angra do Heroísmo \\ 9700-551 - Açores, Portugal \\ E-mail: francisco.simoes@cipp-terceira.com \\ * This work was funded by the Fundação para a \\ Ciência e Tecnologia of the Ministry of Science, \\ Technology and Higher Education of Portugal \\ (SFRH/BD/60823/2009).
}

\begin{abstract}
The present article is a survey of the literature on the effectiveness of school mentoring programs in promoting the socioemotional and instrumental development of youngsters. It starts with a comparison between the most significant empirical investigations on the subject. From such analysis it is possible to observe that the results attributed to mentoring programs are contradictory and tend to be of reduced magnitude. Various methodological weaknesses seem to affect the results obtained in the majority of the works analyzed, amongst which the reduced size of samples and the lack of experimental control of the inherent variability of the subjects. Alongside these methodological limitations, the literature survey carried out here also allowed to identify important variations in the structure of the school mentoring programs, and an uneven weighing of the parameters defining good practices. All these factors seem to make it difficult to obtain solid results about the effectiveness of the kind of school intervention. The recommendations made here for future investigations on this theme include conducting more studies with inferential power about well-structured programs, a greater care in the definition of investigation plans, and the consideration of the teacher as a figure capable of accumulating the function of mentor, contrary to what happens in programs developed in Anglo-Saxon countries.
\end{abstract}

\section{Keywords}

School mentoring - Socioemotional development Instrumental development - Effectiveness. 
A mentoria tem sido encarada, de forma crescente, como uma intervenção válida na promoção do desenvolvimento juvenil. Vários indicadores evidenciam sua legitimação política e institucional, bem como sua progressiva formalização como estratégia destinada ao incremento do desenvolvimento dos jovens. Nos Estados Unidos da América, em 2005, 4.500 agências dedicavam-se ao desenvolvimento de programas de mentoria (KARCHER et al., 2006). Em 2003, estimava-se que 2,5 milhões de jovens norte-americanos estavam enquadrados em programas desse tipo e que outros 15 milhões estariam em condições de beneficiar-se de uma abordagem mentorial. Entre 2003 e 2006, o investimento público em programas de mentoria, nos Estados Unidos, mobilizado por departamentos federais governamentais de áreas tão distintas como a defesa, a educação, a justiça ou a saúde e os serviços sociais, ascendeu a um total de 150 milhões de dólares (KARCHER, 2008). Instituições de referência internacional, como é o caso da Organização Mundial de Saúde, reconhecem-na, também, como uma intervenção útil no quadro da prevenção de comportamentos violentos, a par de outras abordagens relacionais como a terapia familiar ou a educação parental (CHAN; HO, 2008).

$\mathrm{Na}$ base de uma rápida expansão da mentoria está o pressuposto de que a existência de figuras adultas não parentais positivas na vida de crianças e jovens poderá servir como um importante factor promotor de seu desenvolvimento psicossocial (ZIMMERMAN; BINGENHEIMER; NOTTARO, 2002), sobretudo no caso de jovens multiplamente desafiados (DUBOIS et al., 2002). A preponderância dessa premissa surge num contexto sócio-histórico marcado pela crescente inacessibilidade de adultos com semelhantes características, sobretudo em contextos urbanos. Os jovens passam cada vez mais tempo com o grupo de pares, o que reforça um ciclo de organização das relações interpessoais juvenis em que as oportunidades de convivência e partilha com adultos significativos são cada vez mais diminutas (RHODES; REDDY; GROSSMAN, 2005).

0 interesse progressivo pela mentoria tem sido acompanhado, na última década, por um trabalho mais sistemático de investigação científıca, visível não só na proliferação de estudos e publicações sobre os seus mais variados aspectos, mas também no modo como diversas revistas, nas áreas de psicologia, educação e intervenção social, têm dedicado edições especiais à temática. Assiste-se, paralelamente, a uma especialização crescente dos âmbitos de pesquisa e de aplicação da mentoria, a qual se tem traduzido, paulatinamente, num aprofundamento do conhecimento disponivel sobre esse tipo de relação (KARCHER, 2008). Nesse nível, destaca-se o florescimento considerável dos programas e das avaliações sistemáticas sobre mentoria em meio escolar. Trata-se da forma de mentoria em maior expansão, estimando-se que $70 \%$ dos programas de mentoria existentes nos Estados Unidos da América tenham por contexto de aplicação a escola (RANDOLPH; JONHSON, 2008). Não obstante todos esses desenvolvimentos, a investigação mais recente ora tem encontrado benefícios, ora tem detalhado impactos marginais, ou até mesmo nocivos, decorrentes da prática da mentoria em meio escolar (DUBOIS et al., 2002). Ao mesmo tempo, algumas áreas cinzentas permanecem por serem esclarecidas e novas questões vão sendo colocadas, implicando, pois, investigação mais exigente e sistemática.

Neste trabalho, procuraremos clarificar a eficácia de programas de mentoria, aplicados em contexto escolar, no desenvolvimento socioemocional e instrumental do jovem. Após a definição do conceito de mentoria e do enquadramento de suas funções, procederemos à apresentação de um conjunto de seis estudos empíricos e à sua comparação no que diz respeito ao design experimental, método de amostragem aplicado e medidas usadas. Serão, ulteriormente, descritos os principais resultados que tais estudos permitiram compilar. Os mesmos serão analisados 
em referência a outros trabalhos, com especial enfoque para aqueles que definem boas práticas na área da mentoria e, particularmente, da mentoria escolar, por meio da identificação e da tipifıcação de variáveis moderadoras de sua eficácia (DUBOIS et al., 2002; RANDOLPH; JONHSON, 2008). Com esse exercício de revisão crítica da literatura, procuraremos encontrar comunalidades, contradições e limitações da investigação sobre a mentoria desenvolvida em meio escolar, reflectir sobre as condições de implementação que condicionam sua eficácia e extrair implicações que sustentem futuras investigações.

\section{Conceito de mentoria}

A revisão da literatura deixa entrever que o aspecto mais nuclear das diferentes definições de mentoria é sua natureza relacional. Como relação, a mentoria pode ter um início fortuito ou programado e está assente num princípio de desigualdade ou assimetria em que um dos elementos, mormente aquele tido por mais experiente ou conhecedor (o mentor), pode, em certo contexto e durante certo período de tempo, favorecer a consecução de determinado objectivo, normalmente associado ao desenvolvimento do elemento menos experiente (o mentorando) (EBY; RHODES; ALLEN, 2007; KEATING et al., 2002).

Não obstante a dimensão relacional apresentar-se como o eixo unificador da definição de mentoria, registram-se indefinições em vários de seus aspectos cruciais, o que acaba por contribuir para uma pluralidade de significados que acarreta dificuldades não só à sua definição, como à própria investigação empírica (EBY et al., 2007). 0 grau de intimidade que a relação deverá ter, a diferença mínima de idades entre mentor e mentorando, a duração da relação e as funções a serem asseguradas pelo mentor são apenas alguns dos elementos sobre os quais predominam discrepâncias em termos de delimitação conceptual da mentoria (DUBOIS et al., 2002; EBY et al., 2007). Apesar de todas as contradições existentes, parecem existir vectores consensuais na definição do conceito de mentoria, dos quais se salientam: a) o seu cariz pedagógico, pois implica aprendizagem; b) as funções gerais, que compreendem o apoio emocional, a instrumentalidade, no sentido da facilitação na aquisição de competências, e a promoção da autodeterminação do mentorando; c) a organização assimétrica, ainda que potencialmente recíproca da relação; d) a possibilidade de mentor e mentorando beneficiarem-se da relação, embora seu beneficiário primacial seja o mentorando.

Uma vez ponderadas dúvidas e certezas, e apesar do risco inerente a um exercício de síntese, afigura-se-nos como viável definir mentoria como uma relação pessoal em que uma figura adulta fornece orientação, suporte e companhia de forma consistente, com o intuito de desenvolver as competências e a personalidade de uma criança ou de um jovem (MENTOR, 2003 apud KELLER, 2007), no quadro de um referencial cultural comumente aceite e, portanto, passível de transmissão (FREEDMAN, 1993 apud KELLER, 2007).

\section{Funções da mentoria no âmbito do desenvolvimento juvenil}

Grosso modo, a mentoria, como estratégia de promoção do desenvolvimento juvenil, parece favorecer três funções essenciais: apoio socioemocional, suporte instrumental e promoção da autodeterminação.

Assente em certa tese compensatória, inspirada no modelo bioecológico (BRONFENBRENNER; CECI, 1994), e no conceito de resiliência (RUTTER, 2006), a função de apoio socioemocional é, sem dúvida, uma das mais realçadas no âmbito da mentoria, seja ela formal ou informal, promovida em contexto escolar ou comunitário. Os estudos que a ela se reportam visam, sobretudo, compreender os potenciais efeitos da mentoria em jovens que tenham experimentado diversos tipos de dificuldades ou perdas na relação com as figuras parentais ou 
que tenham sido expostos a elevados níveis de adversidade $^{1}$ (DUBOIS et al., 2002; KEATING et al., 2002; KELLER, 2007). Em concreto, procuram compreenderse a mentoria é, em si mesma, um factor de protecção do desenvolvimento juvenil, se ajuda a prevenir ou a minimizar a acção de factores de risco ou se age (in)directamente sobre outros factores protectores (e.g. parentalidade), contribuindo para o ajustamento pessoal do jovem (KELLER, 2005; ZIMMERMAN; BINGENHEIMER; NOTTARO, 2002). Nela também se enquadram os estudos centrados na qualidade da relação, em muito inspirados pela teoria da vinculação (BOWLBY, 1988). Compreender melhor os processos no seio da própria díade mentor/mentorando e seu contributo para a formação de modelos internos de vinculação (in)segura é o objectivo nuclear desta abordagem. Tal análise assenta-se no pressuposto de que os modelos internos são passíveis de revisão em qualquer momento do desenvolvimento pessoal, podendo a mentoria assumir-se, nessa perspectiva, como um contexto favorável para que tal possa suceder.

À mentoria costuma ser também reconhecida uma função instrumental. Esta centra-se em seu carácter pedagógico como veículo promotor de competências, sejam elas pessoais, sociais ou técnicas (KELLER, 2007). Seu estudo tem sido animado pela crescente aceitação de uma visão positiva do desenvolvimento humano e do protagonismo da pessoa na construção de seu próprio conhecimento. Esses pressupostos, ancorados numa perspectiva construtivista do desenvolvimento, têm orientado a investigação no sentido de avaliar o modo como a parceria de aprendizagem entre mentor e mentorando

1. Do ponto de vista do desenvolvimento infantil e juvenil, a designação que mais vezes tem sido usada para descrever esse grupo é a de população em risco. Por população de risco entende-se aquela que acumula uma multiplicidade de desvantagens psicossociais e económicas. № caso de crianças e jovens, as adversidades que costumam ser indicadas como mais relevantes são a vivência em contexto de monoparentalidade, os sinais de perturbação emocional e comportamental, e a ausência de suporte afectivo e instrumental para ultrapassar dificuldades (KEATING et al., 2002). Neste artigo, os jovens nessas condições serão designados como multidesafiados, por considerarmos pertinente contrariar a linguagem e a construção deficitárias que habitualmente Ihes é associada. se operacionaliza. Contudo, inicialmente, o estudo da vertente instrumental da mentoria foi alimentado pela teoria da aprendizagem social e pelos princípios do modelamento (BANDURA, 1977), considerando-se que o mentor era o responsável central pela dinamização do processo de aprendizagem intrínseco à mentoria, emergindo, nessa acepção, como um modelo de referência passível de mimetização. Só posteriormente tal visão foi sendo abandonada em favor de um maior protagonismo do mentorando em sua própria aprendizagem.

A função de autodeterminação (DECI; RYAN, 2008; LARSON, 2006) consiste numa radicalização da centralidade reconhecida ao mentorando no processo de aprendizagem inerente à mentoria e problematiza, ainda que de forma heurística, dois aspectos fundamentais. Por um lado, chama a atenção para o paradoxo da intencionalidade: este consiste no modo como o adulto-, em particular, o mentor- tenta promover a autonomia do jovem de forma prevista e organizada, esquecendo que uma das variáveis cruciais para que tal possa ocorrer é a motivação intrínseca do sujeito e, nesse caso concreto, do mentorando (LARSON, 2006). Por outro lado, e talvez mais importante, ela questiona o facto de a investigação continuar a não avaliar aspectos mais estruturantes da identidade do jovem, como sua autodeterminação, bem como os climas relacionais das relações mentoriais que favorecem um desenvolvimento pessoal a longo prazo (DECI; RYAN, 2000).

Convém ressalvar que embora seja possível distinguir características intrínsecas a cada uma delas, as funções da mentoria não deverão ser entendidas de forma estanque. Em outras palavras, a mesma relação de mentoria poderá servir, simultaneamente, ao apoio socioemocional, à instrumentalidade e à autodeterminação, ainda que enfatize predominantemente uma delas, de forma continuada ou em determinadas fases da relação. Concomitantemente, será de salientar que o estudo dos efeitos das relações mentoriais deverá prever a existência dessas interdependências e a forma como as diferen- 
tes funções da mentoria poderão mutuamente afectar-se, no sentido positivo ou negativo (KARCHER, 2008).

\section{Revisão da literatura}

\section{Metodologia}

A metodologia de revisão de literatura utilizada neste trabalho baseou-se numa consulta às bases de dados de psicologia da EBSCOHOST, realizada em 20 de abril de 2009. Inicialmente, foi feita uma pesquisa genérica de artigos sob a forma de texto completo, com base na palavra-chave mentoring. Foram encontradas 4.721 referências, com datas de publicação compreendidas entre 1958 e 2009. De seguida, limitou-se o intervalo da pesquisa de artigos de texto completo aos últimos dez anos, usando a mesma palavra-chave, tendo sido então encontradas 3.954 referências, o que dá conta da prevalência, na última década, da investigação sobre a temática da mentoria. Finalmente, procedeu-se a um refinamento da pesquisa de referências disponibilizadas, considerando apenas as que surgiam em texto integral e que tinham sido sujeitas a revisão por pares, utilizando os termos school-based mentoring, youth e adolescent development. Nessas condições, foram encontradas 31 referências. Esse exercício de pesquisa permitiu detectar a existência de números especiais de algumas publicações sobre a temática da mentoria, num total de três. Estes foram integralmente adicionados à base de documentos anteriormente constituída, perfazendo um total de 48 referências sobre mentoria e desenvolvimento juvenil. Seguiu-se uma pesquisa complementar às bases de dados da PROQUEST, unicamente centrada nos termos school-based mentoring e adolescent development. Esse segundo exercício de pesquisa teve por intenção alargar a base de programas de mentoria escolar a ser integrada na revisão da literatura, cuja eficácia foi estudada. Foram localizadas mais três referências que se en- contravam nas condições supramencionadas e outros dois artigos que se afiguraram úteis à avaliação crítica do tema.

Do total de 53 referências incluídas numa primeira análise da literatura, 22 eram artigos de investigação empírica, 11 eram artigos de enquadramento teórico, 6 constituíam-se como artigos de revisão da literatura, 3 eram editoriais, outros 3 eram revisões de manuais, 3 eram newsletter, 2 eram capitulos de livros, outros 2 eram artigos publicados em actas de reuniões científicas e 1 era um artigo publicado na imprensa.

\section{Critérios de inclusão para revisão da literatura}

Considerando o enfoque temático deste estudo, foram extraídos seis trabalhos de investigação empírica. Sua selecção baseou-se nos seguintes critérios: a) a investigação deveria estar centrada na eficácia de programas de mentoria desenvolvidos em meio escolar; b) as relações mentoriais estudadas eram de carácter individual, envolvendo mentor e mentorando; c) o mentor deveria ser um adulto; d) os estudos deveriam incluir análises quantitativas dos resultados sobre a eficácia dos programas estudados.

\section{Identificação e breve descrição das investigações revistas}

Os trabalhos incluídos na presente revisão da literatura foram publicados entre $2002 \mathrm{e}$ 2009. 0 mais recentemente publicado é da autoria de Noelle Converse e Benjamin Lingnugaris/ Kraft (2009) e visou estudar o impacto de um programa de mentoria escolar com a duração de 18 semanas, destinado a alunos em risco, com idades compreendidas entre 13 e 15 anos. Como indicadores de inclusão de alunos no programa, foram utilizados como critérios os números de faltas injustificadas e de problemas disciplinares na escola. Os jovens foram encaminhados para o programa de mentoria a partir dos serviços 
de orientação da escola. A produção de impactos sobre aspectos de desempenho escolar era, precisamente, o objectivo central do programa.

Michael Karcher (2008) procurou, igualmente, estudar o impacto de um programa de mentoria escolar no quadro de uma intervenção de enriquecimento académico e social organizado pela Communities in Schools of San Antonio Agency, no estado norte-americano da Califórnia. Nela, a mentoria surgia como uma entre diversas modalidades de intervenção de apoio aos alunos disponibilizadas pela referida agência, sendo activada em conjugação com outro tipo de actividades de apoio/orientação escolar e/ou social. Nesse contexto, a mentoria consistia em encontros semanais de uma hora que se estendiam por um total de oito meses, orientados para alunos com idades que oscilavam entre 10 e 18 anos, os quais se candidatavam espontaneamente ou eram referenciados para inclusão no programa por pais ou professores.

Charles Chan e Wing Ho (2008) estudaram a eficácia do programa de mentoria escolar ICM. Este foi desenvolvido em Hong Kong, tendo sido destinado a adolescentes que frequentavam os três últimos anos do ensino básico e do ensino secundário e que eram considerados em risco por serem oriundos de contextos socioeconómicos marcados pela criminalidade e pela pobreza. 0 programa de mentoria ICM consistia em três ou quatro encontros mensais entre mentor e mentorando, durando a relação mentorial entre 12 e 18 meses. 0 programa procurou enriquecer a vida social e cultural dos jovens, melhorar as suas competências interpessoais, fortalecer o suporte social, favorecer atitudes mais positivas relativamente à educação formal, melhorar relações com pares e pais, e reduzir a ocorrência de comportamentos violentos.

Por seu turno, Leon Dappen e Jody Isernhagen (2006) dedicaram-se ao estudo da eficácia de um programa de mentoria escolar denominado TeamMates. Trata-se de um programa destinado a jovens que frequentam diferentes níveis do ensino básico e secundário. Os jovens aderem de forma espontânea ao programa, não sendo definidos critérios apriorísticos de selecção. A relação mentorial baseia-se em encontros semanais de uma hora, não estando definida, no estudo, sua duração média ou máxima. Os objectivos do programa TeamMates são o estabelecimento de uma relação positiva entre um adulto e um jovem, o desenvolvimento de competências de vida e de interacção, por parte do mentorando, e a facilitação de seu acesso a recursos vários.

Sharon Portwood et al. (2005) investigaram, também, a eficácia de um programa de mentoria escolar intitulado YouthFriends. 0 estudo realizado visou à aplicação desse programa de mentoria em cinco escolas públicas dos estados norte-americanos do Kansas e do Missouri. Tal proposta de mentoria estava aberta a todo o tipo de jovens, com idades compreendidas entre 10 e 18 anos. A relação mentorial baseou-se em encontros semanais de uma hora, ao longo de nove meses. Os jovens foram integrados no programa por meio de sua divulgação com recurso a anúncios, aconselhamento de professores e comunicação informal entre alunos e pais. 0 objectivo central consistiu em incrementar valores e atitudes positivas dos jovens em relação a aspectos como consumo de substâncias, vida escolar, eu e outros significativos, e futuro pessoal.

Finalmente, Keith King et al. (2002) estudaram a eficácia do programa Healthy Kids Mentoring Program. Tratou-se de um programa de mentoria com duração de seis meses destinado a alunos do $4^{\circ}$ ano de escolaridade do sistema de ensino público norte-americano, organizado em torno de quatro componentes essenciais: a) construção da relação; b) melhoria da autoestima do mentorando; c) definição de objectivos; d) assiduidade escolar. Os alunos foram seleccionados por meio de um diagnóstico prévio, baseado num questionário de autor/resposta (55 itens) que cobria aspectos distintos, como autoestima, vinculação à escola, aos pares e à família, e envolvimento em comportamentos de risco. 
Análise e comparação das características metodológicas dos seis estudos revistos

Nesta análise, considerámos três dimensões metodológicas distintas: design do estudo, procedimentos de amostragem e características da amostra e das medidas utilizadas.

No que se refere ao design, dois dos estudos (CONVERSE; LINGNUGARIS/KRAFT, 2009; KARCHER, 2008) são de natureza experimental, procurando controlar a variabilidade atribuída às diferenças entre os sujeitos por meio de procedimentos de distribuição aleatória dos jovens por grupos equivalentes. Adicionalmente, Karcher (2008) recorreu a um método de amostragem probabilística estratificada quanto às variáveis idade e género, nas escolas abrangidas por seu estudo. Em ambos os casos, são estabelecidas comparações pré e pós-teste entre grupos experimental e de controlo. Outros dois estudos utilizam um design quasi-experimental (KING et al., 2002; PORTWOOD et al., 2005) por meio do qual procedem à comparação pré e pós-teste de grupos não equivalentes. Os dois estudos restantes (CHAN; HO, 2008; DAPPEN; ISERNHAGEN, 2006) baseiam-se em designs não experimentais, uma vez que não foram aplicados procedimentos de controlo das diferenças individuais, nem as variáveis independentes foram manipuladas. Nesses casos, foi apenas avaliada a satisfação dos mentorandos, ao final do programa.

Em todos os estudos, as amostras são constituídas por alunos. Alguns estudos consideram, de forma complementar, outros informadores. Converse e Lingnugaris/Kraft (2009) integram 15 mentores em sua investigação, ao passo que Dappen e Isernhagen (2006) incluem 442 professores, 490 pais e 203 mentores. $0 n$ das amostras, no caso dos alunos, varia entre um mínimo de 28 (PORTWO0D et al., 2005) e um máximo de 516 sujeitos (KARCHER, 2008).
No tocante aos métodos de amostragem utilizados, como já foi referido, os estudos de carácter experimental (CONVERSE; LINGNUGARIS/KRAFT, 2009; KARCHER, 2008) utilizaram procedimentos de amostragem probabilística, procedendo à distribuição aleatória dos alunos pelos grupos experimental e de controlo. Todos os estudos restantes utilizaram procedimentos de amostragem não probabilística.

Embora sejam várias as medidas utilizadas nos diversos estudos, elas podem ser, genericamente, enquadradas em duas grandes categorias: medidas socioemocionais e medidas instrumentais (apêndice 1). As primeiras incluem variáveis de natureza cognitiva (e.g. crenças sobre o uso de substâncias, PORTWOOD et al. 2005), afectiva (e.g. depressão, KING et al., 2002), comportamental (e.g. envolvimento em lutas, KING et al., 2002) ou social (e.g. relação com pares, DAPPEN; ISERNHAGEN, 2006). Ao nível instrumental, as medidas encontradas acabam por estar fortemente ligadas ao desempenho escolar (e.g. notas escolares, KARCHER, 2008). Quer se trate da análise de efeitos socioemocionais ou de efeitos instrumentais, será de notar que algumas medidas são analisadas por mais do que um estudo, como é caso da vinculação à escola (CONVERSE; LINGNUGARIS/ KRAFT, 2009; PORTWOOD et al. 2005) ou da autoestima (KARCHER, 2008; KING et al. 2002).

\section{Eficácia dos programas de mentoria escolar: resultados dos estudos}

Resultados ao nível socioemocional

A mentoria parece ter um efeito positivo no incremento de competências comunicacionais e sociais (CHAN; HO, 2008). Programas desse tipo parecem também contribuir para a elevação dos níveis de autoestima dos jovens (KARCHER, 2008; KING et al., 2002), acompanhada por efeitos positivos na ligação a pares e a adultos, incluindo familiares, nomeada- 
mente os pais (CHAN; HO, 2008; CONVERSE; LINGNUGARIS/KRAFT 2009; KARCHER, 2008; KING et al. 2002; PORTWOOD et al., 2005).

Esses resultados atribuídos aos programas de mentoria escolar poderão, todavia, ser moderados por variáveis pessoais como o género ou a idade, nomeadamente no que diz respeito à melhoria da relação com adultos. Karcher (2008), recorrendo a uma amostragem aleatória estratificada de jovens entre 12 e 18 anos, conclui que existem interacções entre a percepção que os jovens têm do efeito da mentoria e as variáveis género e idade. Nesse estudo, fica evidente que são os rapazes, no ensino básico, e as raparigas, no ensino secundário, que reportam maiores benefícios socioemocionais resultantes da participação num programa de mentoria. Um forte investimento dos rapazes na sua autonomia, em fases mais tardias da adolescência, implicando um desafio ao status quo personificado pelo mentor, ou a orientação predominante das raparigas para a intimidade são teses explicativas para os resultados encontrados que são lançadas pelo autor. Outros estudos analisados (CHAN; HO, 2008; DAPPEN; ISERNHAGEN, 2006; KING et al. 2002) não discriminam esse tipo de efeitos de forma estratificada, devido às características das respectivas amostras (centradas numa faixa etária mais concentrada) e ao próprio desenho das investigações (e.g. PORTWOOD et al., 2005) (apêndice 1). Na mesma linha, trabalhos de outros autores (DUBOIS et al., 2002) tendem a corroborar a perspectiva de que a idade não intervém como variável moderadora dos resultados obtidos pela mentoria.

Outra variável que poderá moderar significativamente o impacto da mentoria a nível socioemocional é a qualidade da relação. Entre os pilares que poderão sustentar o desenvolvimento de relações mentoriais de qualidade, Dappen e Isernhagen (2006) destacam o valor central da confiança entre mentor e mentorando. Esse ingrediente relacional é decisivo se se considerar que muitos dos programas de mentoria escolar são concebidos como forma de compensar relacionalmente jovens multidesafiados (CONVERSE; LINGNUGARIS/KRAFT, 2009; KING et al. 2002). Ora, determinados trabalhos indicam que os jovens mais fortemente desafiados pelas suas condições pessoais e sociais, especificamente por experiências prévias de abuso praticadas por adultos, são aqueles que se encontram em maior risco de terminar a relação com um mentor num prazo inferior a três meses, e que relações inferiores a seis meses são potencialmente geradoras de dano para o mentorando (GROSSMAN; RHODES, 2002). Outras investigações acrescentam que a mentoria será uma abordagem relacional contraindicada para jovens com psicopatologia ou sujeitos a experiências negativas prévias com adultos (DUBOIS et al., 2002; KEATING et al., 2002). Cuidados adicionais a ter na indicação de jovens para programas mentoriais, na selecção, formação e supervisão de mentores, e na definição de um processo de matching eficaz são aspectos referidos como boas práticas na implementação de programas de mentoria e que poderão favorecer relações de qualidade (DUBOIS et el., 2002; KARCHER, 2008; RANDOLPH; JONHSON, 2008).

A revisão efectuada permitiu ainda documentar que a mentoria poderá ter uma influência positiva sobre aspectos comportamentais, como a diminuição do número de participações disciplinares (CONVERSE; LINGNUGARIS/ KRAFT, 2009) ou a redução de comportamentos disruptivos em sala de aula (DAPPEN; ISERNHAGEN, 2006). Ao contrário do que tem sido detalhado pela literatura relativamente aos efeitos da mentoria informal (RHODES; REDDY; GROSSMAN, 2005), não foram encontradas diferenças estatisticamente significativas no nível da redução do consumo de substâncias, quando comparados alunos que estavam integrados num programa de mentoria e alunos colocados num grupo de controlo (PORTWOOD et al., 2005). 0 baixo número de alunos que reportavam consumos desse tipo terá condicionado o resultado encontrado, pelo que não será 
adequado, sem mais, rejeitar a hipótese de que a mentoria escolar levará a uma diminuição do consumo de substâncias. Além do processo de amostragem, o conhecimento do mentor sobre essa matéria ou a forma de organização do próprio programa no que se refere à estruturação e ao enfoque das actividades de mentoria, poderão ambos moderar a influência do mentor nessa área (DUBOIS et al. 2002).

\section{Resultados ao nível instrumental}

Os dados analisados sobre a influência da mentoria no desempenho e nos resultados académicos dos mentorandos são contraditórios. King et al. (2002) reportam melhorias genéricas no desempenho escolar após a frequência do programa de mentoria que avaliaram. Em concreto, e com base numa análise dos resultados escolares, $71 \%$ dos alunos tinham melhorado suas notas em pelo menos uma disciplina, em comparação com o período escolar anterior ao início de sua participação no programa. Já 36,4\% dos alunos tinham-no conseguido em duas ou três disciplinas. Chan e Ho (2008) apontam melhorias no desempenho e nas atitudes escolares (especificamente no esforço empregue no trabalho escolar) de 49,6\% dos alunos integrados no programa de mentoria que avaliaram. Os resultados apresentados por esses dois estudos carecem, no entanto, de análises metodologicamente mais refinadas (e.g. comparação pré e pós-teste entre grupos equivalentes). Os dados disponibilizados pelo estudo de Chan e Ho (2008) beneficiariam-se, paralelamente, de uma operacionalização mais clara de certas variáveis, como o desempenho escolar, traduzindo-as em dados objectivos (e.g. notas escolares). A triangulação da informação recolhida junto dos mentorandos com dados fornecidos por outros informadores (e.g. professores) também seria relevante nesse caso.

Portwood et al. (2005) registram, igualmente, melhorias das notas escolares por parte dos alunos abrangidos pelo programa de mentoria escolar que analisaram. Na sequência de terem-no frequentado, os progressos dos jovens são especialmente notórios nos grupos dos rapazes e dos alunos com pior desempenho académico, comparativamente às notas escolares recolhidas previamente à sua integração no programa de mentoria em causa. Contudo, a melhoria nos resultados académicos só é estatisticamente significativa na comparação entre pré e pós-teste no caso dos alunos com piores notas.

Karcher (2008), por seu turno, não recolhe evidências de que a mentoria escolar terá tido influência significativa directa na melhoria das notas dos alunos incluídos no programa que estudou. Os potenciais efeitos são mesmo pequenos quando comparados com os efeitos produzidos pela psicoterapia (WEISZ et al., 1995 apud KARCHER, 2008).

Dappen e Isernhagen (2006) assinalam que embora sejam reconhecidos progressos escolares como resultado da participação no programa de mentoria escolar, uma vez ouvidos mentores, mentorandos, professores e pais, os ganhos evidenciados no plano académico são pequenos. Estes encontram-se mesmo entre as cinco dimensões em que os diferentes informadores reconhecem menor evolução dos alunos após a participação no programa.

Procurando dar alguma inteligibilidade ao impacto instrumental da mentoria escolar, Karcher (2008) propõe um contínuo de influência mútua e circular entre as diferentes funções da mentoria. Concretamente, o autor avança a hipótese de que existirá uma interacção entre alguns efeitos no nível socioemocional e os resultados académicos. Nessa perspectiva, e olhando aos resultados que encontrou, a melhoria em algumas competências pessoais e na ligação com adultos poderá influenciar positivamente o desempenho académico, nomeadamente de rapazes no ensino básico. Trilhando essa linha de raciocínio, e de acordo com investigações anteriores, convém não perder de vista que as discrepâncias na eficácia instrumental de alguns dos programas de mentoria escolar pode- 
rão ser, em parte, explicadas por determinados factores moderadores de natureza temporal, como a frequência e a duração de contactos (DUBOIS et al., 2002). Estes são potencialmente indutores de um reforço circular entre aspectos socioemocionais e instrumentais da mentoria, além de introduzirem aspectos de consistência e de regularidade que estão identificados na literatura como elementos estruturantes de relações próximas (LAURSEN; BUKOWSKI, 1997). Recorde-se que no caso do programa ICM, estudado por Chan e Ho (2008), a interacção entre mentor e mentorando varia de 12 a 18 meses, ao passo que no programa analisado por Karcher (2008) ela não excede oito meses, ainda que a regularidade dos contactos seja sensivelmente a mesma. À luz dos dados oferecidos pela investigação, e não obstante as fragilidades metodológicas particularmente evidentes no estudo de Chan e Ho (2008), diferenças como as que foram encontradas nesses dois trabalhos não deverão ser, pois, ignoradas na leitura do impacto da mentoria nos resultados escolares. Adicionalmente, dados disponíveis sobre a importância da durabilidade de relações de mentoria natural e sua influência nos resultados escolares reiteram a importância desse aspecto na eficácia dos programas mentoriais. Segundo a literatura, as relações com mentores naturais tendem a ser mais duradouras no tempo, e tal facto parece influenciar positivamente o aproveitamento escolar e a manutenção dos jovens na escola, nomeadamente daqueles multiplamente desafiados pelos seus contextos de vida (KLAW; RHODES; FITZGERALD, 2003).

Será ainda de referir que nem todos os estudos revistos abordam o valor instrumental da mentoria. Converse e Lingnugaris/Kraft (2009) apontam como uma das fragilidades de seu trabalho a inexistência de medidas sobre a influência do programa em variáveis como o desempenho ou o abandono escolares. Quando analisadas as investigações com maior atenção, percebe-se que, efectivamente, as hipóteses de trabalho colocam a análise dos possíveis efeitos instrumentais da mentoria num plano secundário, ou então desconsideram-nos como foco de análise. Tal poderá ser atribuído, em parte, ao tipo de programas incluídos nesta revisão, com um enquadramento que legitima a mentoria escolar como um contexto de compensação relacional (e.g., KING et al., 2002) e, por isso mesmo, tendencialmente mais centrado no bem-estar socioemocional.

\section{Limitações gerais dos estudos de eficácia e dos programas de mentoria escolar e implicações para a investigação}

A revisão efectuada permitiu, em primeira instância, identificar um conjunto de limitações metodológicas inerentes aos estudos, discutidas à medida que os seus resultados foram sendo apresentados. Num esforço de sistematização, podemos afırmar que entre elas destacam-se: a) a dimensão pequena de muitas das amostras, limitando, desde logo, a extrapolação das conclusões retiradas; b) a inexistência de controlo experimental da variabilidade pessoal, na maior parte das investigações; c) a dependência excessiva em face de medidas de autorrelato. Reconhecendo essas fragilidades, os autores fazem diversas sugestões de melhoria, entre as quais incluem-se: a) a replicação das respectivas investigações, com base em amostras representativas (CONVERSE; LINGNUGARIS/KRAFT, 2009); b) a utilização de procedimentos que permitam controlar a variabilidade inerente a variáveis pessoais do mentorando (CONVERSE; LINGNUGARIS/KRAFT, 2009); c) a realização de estudos qualitativos que permitam entender, em profundidade, os mecanismos subjacentes às relações mentoriais (KARCHER, 2008; PORTWOOD et al., 2005); d) a compreensão dos efeitos da mentoria escolar a longo prazo, legitimando a necessidade de realizar estudos longitudinais (CONVERSE; LINGNUGARIS/KRAFT, 2009; DAPPEN; ISERNHAGEN, 2006; DUBOIS et al., 2002; KARCHER, 2008); 
e) o recurso a múltiplos informadores, em alternativa à auscultação exclusiva dos mentorandos, na avaliação da eficácia da mentoria escolar (CHAN; HO, 2008).

Além das limitações metodológicas já referidas, a organização dos próprios programas de mentoria escolar tem sido indicada na literatura como um factor que poderá afectar a magnitude e a natureza dos resultados encontrados. Estudos meta-analíticos (DUBOIS et al., 2002) contribuíram para a tipificação das variáveis moderadoras que parecem sustentar intervenções eficazes nessa área, abrindo caminho à sua discussão sistemática em estudos subsequentes. A selecção de mentores, sua formação inicial, a existência de actividades estruturadas de mentoria, a frequência do contacto mentorial, a duração da relação, o envolvimento parental, a monitorização da implementação do programa e a ponderação de mecanismos de compatibilização (matching) entre mentor e mentorando são indicados como condições estruturantes que parecem moderar, de forma mais ou menos significativa, os resultados produzidos por programas de mentoria, tal como, num caso ou noutro, pudemos já constatar.

Detendo-nos nas características dos mentores envolvidos nos programas de mentoria escolar, parece-nos importante discutir um pouco mais sua pertença ou não à escola em que o programa é aplicado. Em muitos casos, estamos perante voluntários, oriundos da comunidade envolvente à escola. Apesar de poder ser invocado o risco da sobreposição de papéis (professor-mentor), há um conjunto de aspectos que, no entanto, reforça a ideia de que os mentores sejam professores, sobretudo quando mantêm um vínculo ao estabelecimento escolar: a) a existência de um background profissional na área da educação parece favorecer uma visão mais positiva de si e da relação (DUBOIS et al., 2002); b) a variação dos climas relacionais de escola para escola faz com que a pertença à instituição possa constituir uma mais-valia; c) o conhecimento dos objectivos e metas dos currículos escolares pode facilitar e reforçar a dimensão instrumental da mentoria escolar (KARCHER, 2008; KELLER, 2007); d) o estabelecimento de relações mentoriais mais duradouras e estáveis (DUBOIS et al., 2002; GROSSMAN; RHODES, 2002).

A organização do tempo escolar em anos lectivos constitui um aspecto que pode afectar, significativa e negativamente, a eficácia da mentoria escolar. É curioso notar que esta se reveste de um cunho desvantajoso, quando comparada com a mentoria informal (KLAW; RHODES; FITZGERALD, 2003; RHODES; REDDY; ROFFMAN, 2005). Nesse particular, Karcher (2008) chega mesmo a afirmar que os resultados da investigação são, de certo modo, surpreendentes, se se tiver em linha de conta que, entre a selecção de mentores, os procedimentos de matching e o início das actividades, os programas de mentoria acabam por ter uma duração que, muitas das vezes, não excede seis meses. A organização dos programas antes do início das aulas poderia ajudar a contornar essa dificuldade e a aumentar o tempo útil da relação.

A redução das actividades de mentoria a um tempo estanque no horário e a exercícios de caneta e papel, tal como surge nos estudos revistos, parece inadequada quando se pretende promover um contexto de proximidade relacional e de confiança. A possibilidade de aumentar os níveis de informalidade da relação e de disponibilidade do mentor pode favorecer uma maior proximidade entre mentor e mentorando, e, consequentemente, a concretização dos objectivos da mentoria escolar com maior eficácia (CHAN; HO, 2008; LAURSEN; BUKOWSKI, 1997). Estudos adicionais e de natureza exploratória acerca dos factores promotores da qualidade relacional são, pois, desejáveis e genericamente recomendados (DUBOIS et al., 2002; KARCHER, 2008; PORTWOOD et al., 2005). 
A narrativa de défice que acompanha a implementação de muitos programas de mentoria escolar constitui uma categoria distinta de dificuldades que consideramos também importante sublinhar. Em alguns dos estudos revistos, os alunos são designados para a frequência do programa mentorial pelo risco psicossocial ou por dificuldades de aprendizagem ou de comportamento que apresentam (CONVERSE; LINGNUGARIS/ KRAFT, 2009; KING et al., 2002). Os programas são, pois, selectivos e decorrem de um princípio correctivo ou de controlo social que poderá dificultar a adesão à relação mentorial. No extremo, a inclusão de jovens tendencialmente mais perturbados do ponto de vista emocional e/ou comportamental poderá fazer a mentoria tender para um espaço de natureza psicoterapêtica que, de todo, não pode assumir (DUBOIS et al., 2002). A possibilidade de estudar o cruzamento de uma dimensão individual da mentoria com outras modalidades (e.g. mentoria de grupo), dirigidas, de forma integrada, a grupos não conotados com uma narrativa de problema poderá esclarecer se tal princípio de organização ajudará a dar outro sentido à mentoria, a normalizar esse tipo de resposta e a favorecer sua eficácia.

Finalmente, não podemos deixar de sublinhar que, para além da relevância secundária dada à função instrumental, a avaliação da mentoria escolar em dimensões mais ligadas ao desenvolvimento identitário do jovem e à sua autodeterminação, tal como tem sido proposto na literatura, é inexistente nos estudos revistos (DECI; RYAN, 2008; LARSON, 2006). 0 reconhecimento das várias dificuldades de organização e normalização dos programas, com consequente limitação da intensidade, duração e estabilidade das relações, poderá justificar essa aparente omissão.

\section{Conclusões}

A revisão da literatura permite verificar que existe um corpo crescente, mas ainda reduzido, de investigação empírica dedicada ao estudo da eficácia dos programas de mentoria escolar. Tal parece responder a um incremento desse tipo de resposta. Todavia, os estudos denotam, ainda, diversas limitações. Por um lado, surgem fragilidades metodológicas (e.g. reduzido número de trabalhos de carácter experimental, tamanho limitado das amostras) para as quais várias soluções vêm já sendo apontadas. Por outro lado, a organização dos programas carece de ajustamentos variados, entre os quais se destacam a garantia de condições de durabilidade e estabilidade das relações e a desmistificação da mentoria como uma resposta compensatória e/ou de controlo social para jovens-problema ou em risco.

Em face dessas limitações, os estudos sobre a eficácia da mentoria escolar apresentam resultados contraditórios ou de uma magnitude reduzida, quer no que se refere ao desenvolvimento de aspectos socioemocionais, quer no tocante ao desenvolvimento de dimensões instrumentais. A instrumentalidade da mentoria escolar parece ocupar um lugar secundário, em particular no que diz respeito aos resultados escolares, e os efeitos sobre a potencial função de autodeterminação do jovem são mesmo inexistentes.

Apesar dessas dificuldades, uma preocupação crescente dos programas de mentoria escolar em integrar os princípios definidores de boas práticas (DUBOIS et al, 2002), associada a estudos com poder inferencial, poderá ajudar a esclarecer, no futuro, qual é o potencial efectivo de programas dessa natureza na promoção do desenvolvimento psicossocial de jovens. 


\section{Referências}

BANDURA, A. Social learning theory. New York: General Learning Press, 1977.

BOWLBY J. A secure base: clinical applications of attachment theory. London: Routledge, 1988.

BRONFENBRENNER, U.; CECI, S. J. Nature vs. nurture in developmental perspective: a bioecological model. Psychological Review, v. 101, n. 4, p. 568-586, 1994.

CHAN, C.; HO, W. C. An ecological framework for evaluating relationship-functional aspects of youth mentoring. Journal of Applied Social Psychology, v. 38, n. 4, p. 837-867, 2008.

CONVERSE, N.; LINGNUGARIS/KRAFT, B. Evaluation of a school-based mentoring program for at-risk middle school youth. Remedial and Special Education, v. 30, n. 1, p. 33-46, 2009.

DAPPEN, L.; ISERNHAGEN, J. D. Urban and nonurban schools: examination of a statewide mentoring program. Urban Education, v. 41, n. 2 , p. $151-168,2006$.

DECI, E. L.; RYAN, R. M. The "what" and "why" of goal pursuits: human needs and the self-determination of behavior. Psychological Inquiry, v. 11, n. 4, p. 227-268, 2000.

. Self-determination theory: a macrotheory of human motivation, development, and health. Canadian Psychology, v. 49, n. 3, p. 182-185, 2008.

DUBOIS, D. L. et al. Effectiveness of mentoring programs for youth: a meta-analytic review: American Journal of Community Psychology, v. 30, n. 2, p. 157-197, 2002.

EBY, L.; RHODES, J. E.; ALLEN, T. Definition and evolution of mentoring. In: ALLEN, T.; EBY, L. (Orgs.). The blackwell handbook of mentoring: a multiple perspectives approach. Malden: Blackwell Publishing, 2007.

GROSSMAN, J.; RHODES, J. E. The test of time: predictors and effects of duration in youth mentoring relationships. American Journal of Community Psychology, v. 30, n. 2, p. 199- 219, 2002.

KARCHER, M. The study of mentoring in the learning environment (SMLEE). a randomized evaluation of the effectiveness of schoolbased mentoring. Prevention Science, v. 9, n. 2, p. 99-113, 2008.

KARCHER, M. et al. Mentoring programs: a framework to inform program development, research and evaluation. Journal of Community Psychology, v. 34, n. 6, p. 709-725, 2006.

KEATING, L. M. et al. The effects of mentoring program on at-risk youth. Adolescence, v. 37, n. 148, p. 717-734, 2002.

KELLER, T. A systemic model of the youth mentoring intervention. The Journal of Primary Prevention, v. 26, n. 2, p. 169-188, 2005. . Youth mentoring: Theoretical and metohodological issues. In: ALLEN, T.; EBY, L. (Orgs.). The blackwell handbook of mentoring: a multiple perspectives approach. Malden: Blackwell Publishing, 2007.

KING, K. et al. Increasing self-esteem and school connection trough a multidimensional mentoring program. The Journal of School Health, v. 72, n. 7, p. 294-299, 2002.

KLAW, E.; RHODES, J. E., FITZGERALD, L. Natural mentors in the lives of african american adolescent mothers: tracking relationships over time. Journal of Youth and Adolescence, v. 32, n. 2, p. 223-232, 2003.

LARSON, R. Positive youth development, willfull adolescents and mentoring. Journal of Community Psychology, v. 34, n. 6, p. 677-689, 2006. 
LAURSEN, B.; BUKOWSKI, W. A developmental guide to the organization of close relationships. International Journal of Behavioral Development, v. 21, n. 4, p. 747-770, 1997.

PORTWOOD, S. G. et al. YouthFriends: Outcomes from a school-based mentoring program. The Journal of Primary Prevention, v. 26, n. 2, p. 129-145, 2005.

RANDOLPH, K. A.; JOHNSON, J. L. School-based mentoring: a review of the research. Children \& Schools, v. 30, n. 3, p. 177-185, 2008.

RHODES, J. E.; REDDY, R.; GROSSMAN, J. B. The protective influence of mentoring on adolescents' substance use: direct and indirect pathways. Applied Developmental Science, v. 9, n. 1, p. 31-47, 2005.

RUTTER, M. The promotion of resilience in the face of adversity. In: A. CLARKE-STEWART, A.; DUNN, J. (Orgs.). Families count: effects on child and adolescent development. New York: Cambridge University Press, 2006.

ZIMMERMAN, M. A.; BINGHENHEIMER, J. B.; NOTARO, P. C. Natural mentors and adolescent resiliency: a study with urban youth. American Journal of Community Psychology, v. 30, n. 2, p. 221-243, 2002.

Recebido em 28.01.10

Aprovado em 09.09.10

Francisco Simões é doutorando em Psicologia Clínica da Família pelas Universidades de Coimbra e de Lisboa, e bolseiro da Fundação para a Ciência do Ministério da Ciência e Ensino Superior de Portugal.

Madalena Alarcão é professora associada da Faculdade de Psicologia e de Ciências da Educação da Universidade de Coimbra. E-mail: malarcao@fpce.uc.pt. 
Apêndice 1 - Análise sumária dos aspectos metodológicos dos estudos revistos

\begin{tabular}{|c|c|c|c|}
\hline Estudo & Design & $\begin{array}{c}\text { Amostragem e } \\
\text { tamanho da amostra }\end{array}$ & Medidas utilizadas \\
\hline CHAN; HO (2008) & $\begin{array}{l}\text { Multimetodológico } \\
\text { sequencial, incluindo } \\
\text { um estudo quantitativo } \\
\text { não experimental: } \\
\text { questionário aplicado aos } \\
\text { mentorandos pós-teste, } \\
\text { seguido de entrevista em } \\
\text { profundidade. }\end{array}$ & $\begin{array}{l}\text { Não probabilística: } \\
106 \text { alunos no estudo } \\
\text { quantitativo; } 48 \text { alunos } \\
\text { no estudo qualitativo, } \\
\text { seleccionados } \\
\text { aleatoriamente a partir da } \\
\text { amostra inicial. }\end{array}$ & $\begin{array}{l}\text { Ao nível contextual: características da relação; } \\
\text { características do programa; características da escola } \\
\text { e da comunidade; ao nível pessoal: características do } \\
\text { mentor e do mentorando; eficácia do programa: em } \\
\text { dimensões como enriquecimento social e cultural, } \\
\text { competências comunicacionais e sociais, apoio } \\
\text { social, desempenho académico, relação com pares, } \\
\text { relação com pais e comportamento pró-social. }\end{array}$ \\
\hline $\begin{array}{l}\text { DAPPEN; } \\
\text { ISERNHAGEN (2006) }\end{array}$ & $\begin{array}{l}\text { Não experimental: } \\
\text { inquérito pós-teste } \\
\text { aplicado a alunos, } \\
\text { professores e mentores. }\end{array}$ & $\begin{array}{l}\text { Não probabilística: } 501 \\
\text { alunos, } 442 \text { professores, } \\
490 \text { pais e } 203 \text { mentores. }\end{array}$ & $\begin{array}{l}\text { Relação com pares; competências pró-sociais; } \\
\text { comportamento na sala de aula; envolvimento } \\
\text { académico; aspirações futuras; autoconfiança. }\end{array}$ \\
\hline KARCHER (2008) & $\begin{array}{l}\text { Experimental: aplicação } \\
\text { pré e pós-teste da bateria } \\
\text { de instrumentos, a } \\
\text { grupos equialentes. }\end{array}$ & $\begin{array}{l}\text { Amostragem probabilística } \\
\text { estratificada (por género } \\
\text { e nível escolar): } 516 \text { alunos. }\end{array}$ & $\begin{array}{l}\text { Ligação emocional, autoestima, percepção de suporte } \\
\text { social, competências sociais, esperança, percepção } \\
\text { de interesse por parte dos outros, notas escolares. }\end{array}$ \\
\hline KING et. al. (2002) & $\begin{array}{l}\text { Quasi-experimental: pré } \\
\text { e pós-teste; comparação } \\
\text { de grupos não } \\
\text { equivalentes. }\end{array}$ & $\begin{array}{l}\text { Não probabilística: } 28 \\
\text { alunos seleccionados com } \\
\text { base em sua caracterização } \\
\text { como estando em situação } \\
\text { de risco de uma população } \\
\text { de } 283 \text { alunos (grupo de } \\
\text { controlo). }\end{array}$ & $\begin{array}{l}\text { Autoestima; ligação à escola, aos pares e à família; } \\
\text { bullying; uso de substâncias; envolvimento em lutas: } \\
\text { depressão. }\end{array}$ \\
\hline $\begin{array}{l}\text { PORTWOOD et. al. } \\
\text { (2005) }\end{array}$ & $\begin{array}{l}\text { Quasi-experimental: pré } \\
\text { e pós-teste; comparação } \\
\text { de grupos não } \\
\text { equivalentes. }\end{array}$ & $\begin{array}{l}\text { Não probabilística: } 170 \\
\text { alunos no pós-teste. }\end{array}$ & $\begin{array}{l}\text { Conhecimento, crenças e uso de substâncias; } \\
\text { atitudes em relação à escola; atitudes em relação a si } \\
\text { próprio, aos adultos e ao futuro; vinculação à escola; } \\
\text { desempenho escolar. }\end{array}$ \\
\hline $\begin{array}{l}\text { CONVERSE; } \\
\text { LIGNUGARIS/KRAFT } \\
\text { (2009) }\end{array}$ & $\begin{array}{l}\text { Experimental: inquérito } \\
\text { pré e pós-teste a } \\
\text { mentorandos e mentores } \\
\text { sobre atitudes face à } \\
\text { escola. Comparação entre } \\
\text { grupos equivalentes. }\end{array}$ & $\begin{array}{l}\text { Amostragem probabilística: } \\
32 \text { alunos e } 15 \text { mentores. }\end{array}$ & $\begin{array}{l}\text { Número de referências disciplinares por aluno } \\
\text { registadas em seu processo individual; número de } \\
\text { faltas injustificadas por aluno; atitudes perante a } \\
\text { escola (autoestima, ligação aos pares e vinculação } \\
\text { à escola) }\end{array}$ \\
\hline
\end{tabular}

